\title{
Spatial spreading of magnetospherically reflected chorus elements in the inner magnetosphere
}

\author{
H. Breuillard ${ }^{1}$, Y. Zaliznyak ${ }^{2}$, O. Agapitov ${ }^{1,3}$, A. Artemyev ${ }^{1,4}$, V. Krasnoselskikh ${ }^{1}$, and G. Rolland ${ }^{5}$ \\ ${ }^{1}$ LPC2E/CNRS-University of Orléans, UMR7328, Orléans, France \\ ${ }^{2}$ Institute for Nuclear Research, Kyiv, Ukraine \\ ${ }^{3}$ National Taras Shevchenko University of Kyiv, Kyiv, Ukraine \\ ${ }^{4}$ Space Research Institute, RAS, Moscow, Russian Federation \\ ${ }^{5} \mathrm{CNES}$, Toulouse, France \\ Correspondence to: H. Breuillard (hbreuill@gmail.com)
}

Received: 12 March 2013 - Revised: 22 May 2013 - Accepted: 19 July 2013 - Published: 26 August 2013

\begin{abstract}
Chorus-type whistler waves are known to be generated in the vicinity of the magnetic equator, in the lowdensity plasma trough region. These wave packets propagate towards the magnetic poles, deviating from the magnetic field lines, before being eventually reflected at higher latitudes. Magnetospheric reflection of whistler waves results in bounce oscillations of these waves through the equator. Our study is devoted to the problem of geometrical spreading of these whistler-mode waves after their first magnetospheric reflection, which is crucial to determine where wave-particle interactions occur. Recently, experimental studies stated that the relative intensity of the reflected signal was generally between 0.005 and 0.05 of the source signal. We model such wave packets by means of ray tracing technique, using a warm plasma dispersion function along their trajectory and a realistic model of the inner magnetosphere. We reproduce the topology of the reflected energy distribution in the equatorial plane by modeling discrete chorus elements generated at the equator. Our calculations show that the spatial spreading is large and strongly dependent upon initial wave parameters, especially the chorus wave frequency. Thus, the divergence of each element ray trajectories can result in the filling of a large region (about 4 Earth radii around the source) of the magnetosphere and a reflected intensity of $0.005-0.06$ of the source signal in the equatorial plane. These results are in good agreement with previous Cluster and THEMIS observations.
\end{abstract}

Keywords. Radio science (magnetospheric physics)

\section{Introduction}

Chorus-type whistler waves are one of the most intense natural electromagnetic emissions observed in the inner magnetosphere. They have been intensively studied up to now (see, e.g., comprehensive reviews by Sazhin and Hayakawa, 1992; Trakhtengerts and Rycroft, 2008, and references therein), because they are critical to electron acceleration and losses (Summers et al., 2002; Horne et al., 2005; Summers et al., 2007; Shprits et al., 2008; Hikishima et al., 2010; Santolík et al., 2010), which define the Earth's radiation belt dynamics (Lyons and Thorne, 1973). Magnetospheric chorus waves typically appear as short coherent wave bursts propagating in the non-ducted whistler mode in two distinct frequency bands, scaling on the equatorial electron gyrofrequency $\Omega_{\mathrm{e}, \mathrm{eq}}$. The lower-band frequency range is known to be $\omega \approx 0.15-0.45 \Omega_{\mathrm{e}, \mathrm{eq}}$, and the upper-band frequency range is $\omega \approx 0.5-0.7 \Omega_{\mathrm{e}, \mathrm{eq}}$ (see, e.g., Burtis and Helliwell, $1969,1976)$, the latter being substantially less intense than the lower-band chorus (Meredith et al., 2001; Haque et al., 2010).

Chorus waves are usually observed in the midnight, dawn and noon sectors (Agapitov et al., 2011a; Meredith et al., 2003; Li et al., 2009; Meredith et al., 2012) with a maximum in occurrence rate and wave intensity between 06:00 and 12:00 MLT (see, e.g., Li et al., 2009; Agapitov et al., 2011a) for rather quiet magnetospheric conditions $(\mathrm{Kp} \leq 4)$. The chorus source region is known to be located in the vicinity of the magnetic equator (see LeDocq et al., 1998; Santolík et al., 2005b,a) for the vast majority of these waves, 

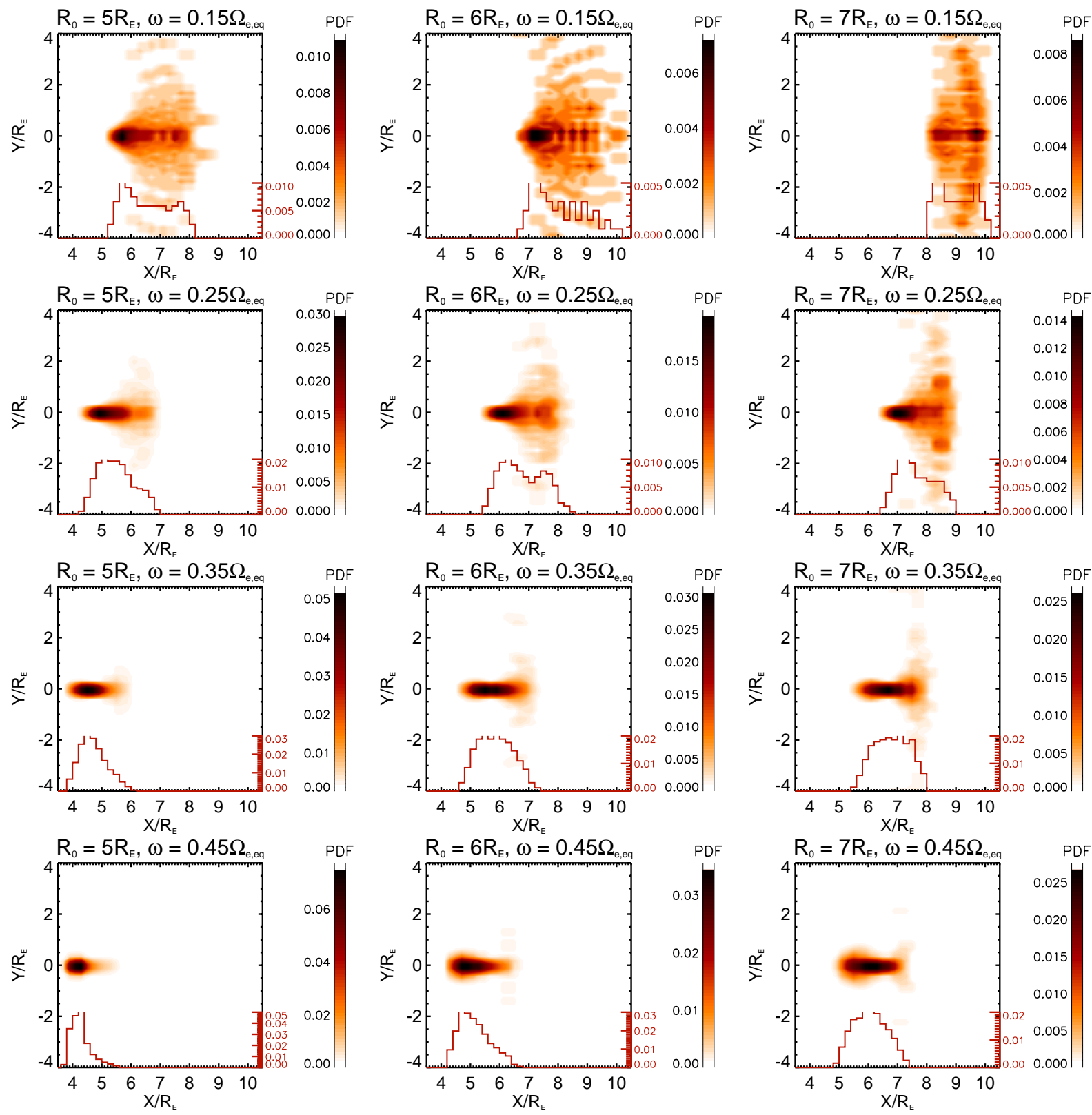

Fig. 1. The reflected wave power of selected "cones" of rays in the equatorial plane, after their first reflection at high latitudes. Each cone of rays consists of one selected frequency $\omega$ and one starting point $R_{0}$, with polar and azimuthal wave normal angles in the ranges $0^{\circ} \leq \theta_{0} \leq 40^{\circ}$ and $-180^{\circ} \leq \varphi_{0} \leq 180^{\circ}$, respectively. The histogram (red) shows the cross section of the distribution along the $x$ axis for $Y \approx 0$.

although sometimes the source can be also situated in nonequatorial minimum B-pockets during high magnetospheric activity (Tsurutani and Smith, 1977). They are also known to be generated outside the plasmasphere with the amplitude maximum at $L \approx 7$ (Meredith et al., 2003; Santolík et al., 2005a; Li et al., 2009; Meredith et al., 2012) during the injection of highly anisotropic particle populations into the radiation belts. Experimental studies onboard different spacecraft (see Burton and Holzer, 1974; Hayakawa et al., 1986; Agapitov et al., 2011a; Li et al., 2011, for instance) have shown that the wave normal distribution of intense chorus waves (i.e., lower-band, rising elements) is generally non-Gaussian with a non-zero mean value. These effects are enhanced as magnetic latitude increases.

As whistler waves propagate away from the magnetic equator, their wave normal angle increases and they can be trapped within Earth's inner magnetosphere. These waves can indeed be magnetospherically reflected at high latitudes (e.g., Thorne and Kennel, 1967; Lyons and Thorne, 1970; Jirícek et al., 2001), where the local lower hybrid frequency 
is likely to become higher than the wave frequency (see Shklyar et al., 2004, for details). If so, wave propagation at an arbitrary angle is allowed (Hines, 1957), and the wave normal angle can become obtuse. The wave is thereby "reflected" at its mirror point and propagates back to the equator. This phenomenon is known as the lower hybrid resonance (LHR) reflection. Non-ducted whistler waves can thus perform several bounces between their mirror points before eventually being completely damped (see, e.g., Kimura, 1966; Chum and Santolík, 2005; Bortnik et al., 2008, 2009, and references therein).

Multiple reflection of whistler waves has been first demonstrated by ray tracing simulations (Kimura, 1966). Spacecraft observations of magnetospherically reflected whistler waves were then discussed by Edgar and Smith (1967) and Smith and Angerami (1968) for example. However, experimental evidence of reflected chorus waves at lower latitudes is scarce due to the fact that calculations of Poynting vector have been performed only recently. The first observations of these reflected wave packets were presented by Parrot et al. $(2003,2004)$ using Cluster spacecraft multi-point measurements, showing simultaneous observations of the source and reflected whistler chorus by the STAFF-SA instrument. A similar study, combining THEMIS observations and ray tracing simulations, has been performed by Agapitov et al. (2011b), explaining the characteristics of observed reflected waves by the divergence of their trajectories (see also Chum and Santolík, 2005).

Making use of the same numerical model as in Agapitov et al. (2011b), which is described in detail in Breuillard et al. (2012b), we perform in the present paper a study of the spatial spreading of magnetospherically reflected waves, by comparing the distribution of numerous trajectories of rays with previous results from Cluster (Parrot et al., 2003, 2004) and THEMIS (Agapitov et al., 2011b) spacecraft. This divergence of ray trajectories allows one to determine the wave power distribution of chorus emissions, and thereby to estimate where wave-particle interactions occur in the inner magnetosphere.

In the following sections we shall first describe the topology of reflected chorus wave power in the equatorial plane, with respect to the ray initial parameters. Then we present the methodology to model discrete chorus elements, and their reflected wave power distribution at the equator. The obtained intensity of reflected chorus emissions, relative to their source signals, is then compared to previous experimental and theoretical results. Finally, we present a discussion and some conclusions.

\section{Topology of reflected chorus wave power in the equatorial plane}

In Agapitov et al. (2011b), a set of observed chorus waves generated at the magnetic equator was propagated in the inner magnetosphere by means of three-dimensional ray tracing technique. The distribution of these rays intersecting the equatorial plane after their first magnetospheric reflection was notably presented. In this section we perform a similar analysis, but with a greatly extended set of rays. We use here the trajectories of $\sim 60000$ numerical rays representing most of the chorus parameters observed in the inner magnetosphere, which allows us to study statistically their wave power distribution after their reflection at high latitudes. The wave power is calculated in this study as the number of waves that cross a unite area, because waves are propagated in thermal plasma, and thus the wave amplitude variations are negligible.

We model chorus waves in the Earth's magnetosphere using ray tracing technique (i.e., in the frame of WentzelKramers-Brillouin (WKB) approximation), which implies that the characteristic scale of the medium property variation is larger than the wavelength of the chorus wave. This assumption here is valid since the wavelength of such waves is approximately tens of kilometers while the scale of the global density and magnetic field changes is about one Earth radius. Realistic models of core plasma densities (Gallagher et al., 2000) and magnetic field (Olson and Pfitzer, 1977), as well as ray tracing procedure (Suchy, 1981), are employed in our numerical code, which is described in detail in Breuillard et al. (2012b). We also use the same database of ray trajectories as in Breuillard et al. (2012b), but with an extended range of wave frequencies (step is $0.05 \Omega_{\mathrm{e}, \mathrm{eq}}$ starting from $0.1 \Omega_{\mathrm{e}, \text { eq }}$ to $0.5 \Omega_{\mathrm{e}, \mathrm{eq}}$, where $\Omega_{\mathrm{e} \text {,eq }}$ is the equatorial electron gyrofrequency), which represents the entire lower-band chorus source region.

The trajectory of a whistler wave propagating in the inner magnetosphere strongly depends on its initial parameters, in particular its frequency $\omega$ (normalized to $\Omega_{\mathrm{e}, \mathrm{eq}}$ ) and starting point $R_{0}$ in the equatorial plane. In each panel of Fig. 1, we show the wave power distribution of a set of rays in the equatorial plane, resulting from its first magnetospheric reflection, with respect to its initial parameters. The selected set of rays launched away from equator consists of rays of one given frequency $\omega$ at one starting point $R_{0}$ (not shown) with coordinates $\left[X_{0} / R_{\mathrm{E}}, 0,0\right]$. At the equator, rays in each set have initial polar wave normal angles in the range $0^{\circ} \leq \theta_{0} \leq 40^{\circ}$ as it is commonly observed in the equatorial region (see, e.g., Agapitov et al., 2012). Rays' initial azimuthal wave normal angles are in the range $-180^{\circ} \leq \varphi_{0} \leq 180^{\circ}$. Thus a set is three-dimensional and represents a cone of about 150 ray trajectories.

Figure 1 firstly shows that the divergence of reflected ray trajectories strongly depends on the wave frequency. The deviation in $L$ shell and longitude from their initial magnetic field line is indeed much larger for lower frequencies $\left(\omega \leq 0.3 \Omega_{\mathrm{e}, \mathrm{eq}}\right)$, and results in a spread wave power distribution in the equatorial plane. Lower frequency waves are preferably deviated towards larger $L$ shells. Their spreading can reach $\sim 4 R_{\mathrm{E}}$ in both radial and azimuthal distance. On 


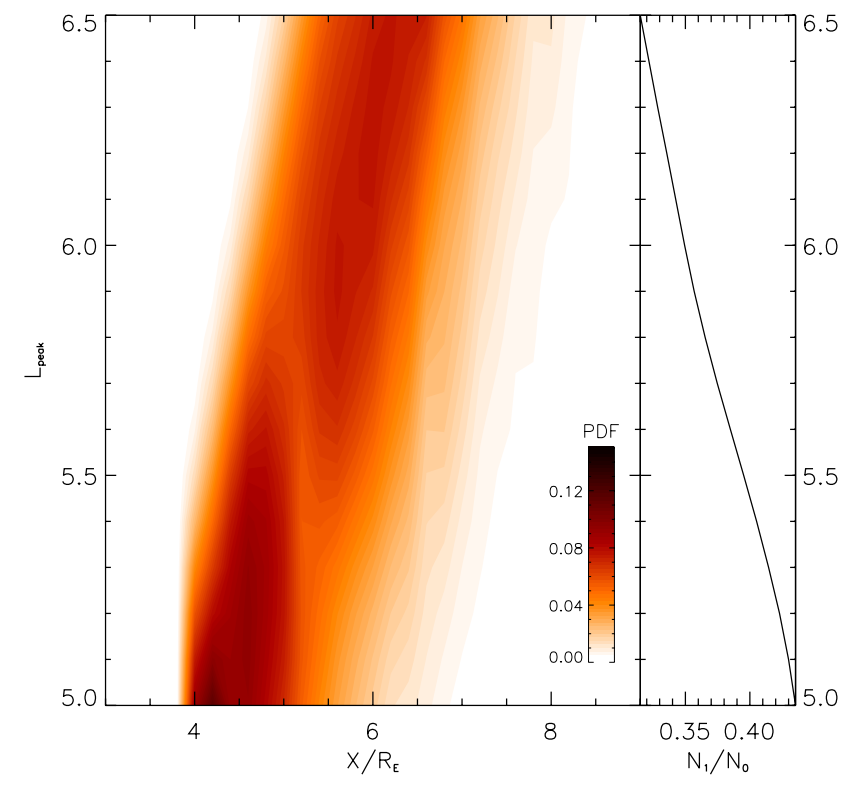

Fig. 2. The reflected wave power of a single chorus element in the equatorial $X Y$ plane as a function of radial distance ( $x$ axis), for different values of $L_{\text {peak }}$ ( $y$ axis). Each distribution (line) is obtained from an initial single chorus element generated at the equator at $Y=0$, centered at $L_{\text {peak }}$, and intensity-weighted as in Eq. (1). The value of the distribution in each bin (of $0.2 R_{\mathrm{E}}$ width) is shown in the color bar. The ratio of the number of reflected rays (that effectively reach the equator) over the initial number of rays in each single element is shown on the right panel, as a function of $L_{\text {peak }}$ value.

the contrary, higher frequency waves $\left(\omega \geq 0.3 \Omega_{\mathrm{e}, \mathrm{eq}}\right)$ rather propagate towards lower $L$ shells and their distribution is much more focused around $Y=0$. The divergence of ray trajectories also depends on their starting point $R_{0}$, though it is less pronounced than their dependence on frequency. The wave power distribution is generally more spread for larger $R_{0}$ as rays travel a larger distance throughout the inner magnetosphere. However, we see that the major part of the distribution is concentrated around the rays' original longitude $(Y=0)$, especially for higher frequency waves, as shown on the $x$ axis by the histogram in red. This histogram is obtained by processing the cross section of the 2-D distribution along the $x$ axis for $Y= \pm 0.2$.

This distribution (in red) can be used to compare the attenuation, relative to its source signal, of such reflected wave power with spacecraft data. However it is necessary first to model discrete chorus elements properly.

\section{Reflected wave power distribution of chorus elements at the equator}

A single chorus element (denoted hereafter SCE) can be modeled by a group of waves generated over a $L$ shell region of $\sim 1 R_{\mathrm{E}}$ (step is $0.1 R_{\mathrm{E}}$ ) with a wave power distribution approximated by a Gaussian function. For the considered MLT and Kp parameters (i.e., $\mathrm{Kp}=4$ and MLT =09:00; see Breuillard et al., 2012b), the chorus wave normal distribution is presented in Agapitov et al. (2012) (see also Agapitov et al., 2013) from which we obtain a function $h_{0}\left(\theta_{0}\right)$ representing the distribution of $\theta_{0}$ angles at the equator. The distribution in frequency is also approximated by a Gaussian function (see, e.g., Breuillard et al., 2012b), so that the distribution of rays is intensity-weighted by the following function:

$$
\begin{aligned}
g_{0}\left(\theta_{0}, L_{0}, \omega\right)= & h_{0}\left(\theta_{0}\right) \exp \left[-\frac{\left(L_{0}-L_{\text {peak }}\right)^{2}}{2(0.2)^{2}}\right] \\
& \times \exp \left[-\frac{\left(\omega-0.34 \Omega_{\mathrm{e}, \mathrm{eq}}\right)^{2}}{2\left(0.15 \Omega_{\mathrm{e}, \mathrm{eq}}\right)^{2}}\right] .
\end{aligned}
$$

In our case each SCE is launched at MLT = 09:00 (see Breuillard et al., 2012b,a), i.e., $Y_{0}=0$ for all rays. Core plasma density and magnetic field parameters are the same as used in Breuillard et al. (2012b). A SCE is modeled here by $\sim 20500$ rays, with parameters corresponding to commonly observed lower-band chorus waves. The frequency range is $0.15 \Omega_{\mathrm{e}, \mathrm{eq}} \leq \omega \leq 0.45 \Omega_{\mathrm{e}, \mathrm{eq}}$ with a step of $0.05 \Omega_{\mathrm{e}, \mathrm{eq}}$. The azimuthal angle range of $\boldsymbol{k}$ vector is $-180^{\circ} \leq \varphi_{0} \leq+180^{\circ}$ (step is $10^{\circ}$ ), whereas $\theta_{0}$ lies in the range $0^{\circ} \leq \theta_{0} \leq 40^{\circ}$ (step is $5^{\circ}$ ).

It is then possible to model the reflected wave power distribution of such SCE in the equatorial $X Y$ plane. We record the distribution of reflected waves at the same longitude as their starting point, i.e., at $Y=0$ (see histogram in Fig. 1), as it is observed onboard spacecraft during simultaneous measurements of source and reflected signals (Parrot et al., 2004; Agapitov et al., 2011b). We study the dependence of the reflected wave power on $L$ shell for different $L_{\text {peak }}$ values.

This dependence is presented in Fig. 2, where the equatorial wave power distribution over $L$ shells is shown on the $x$ axis, as a function of the initial radial distance of the SCE source. The latter is represented by the radial distance $L_{\text {peak }}$ of the peak of each SCE, in $R_{\mathrm{E}}$ on the $y$ axis. On the right panel the ratio of the global reflected wave power over the initial wave power at the starting point of each SCE is plotted (i.e., the number $N_{1}$ of reflected waves that effectively reach the equatorial plane over the initial number $N_{0}$ of waves).

First, one can note that the wave power is more spread over $L$ shells for higher values of $L_{\text {peak }}$, even though the maximum of distribution is still shifted by about $0.5 R_{\mathrm{E}}$ towards lower $L$ shells. For lower values of $L_{\text {peak }}$ (below $\sim 6 R_{\mathrm{E}}$ ), the wave power is concentrated just beyond the plasmapause 
(here the plasmapause is situated at $4 R_{\mathrm{E}}$ in the equatorial plane). In particular, for $L_{\text {peak }} \leq 5.2$ the distribution of the wave power is narrow (its maximum value reaches $\sim 0.15$ ) and close to the plasmapause, but it drops abruptly for distances below this value. This means that most of the waves are accumulated at the plasmapause and cannot penetrate into the plasmasphere. On the right panel the ratio $N_{1} / N_{0}$ of reflected waves is found to be decreasing with increasing $L_{\text {peak }}$ (from $\sim 42 \%$ to $\sim 32 \%$ ).

Then it is possible to calculate the wave power of reflected rays, compared to the initial SCE wave power. Indeed, the multiplication of these two ratios for each bin thus gives the attenuation ratio of reflected waves over a distance of $0.2 R_{\mathrm{E}}$, which allows us to compare this value to the attenuation ratio obtained previously by observations and theoretical calculations. Figure 2 shows that this attenuation ratio value varies from about 0.005 for the regions where the least waves are reflected to 0.06 for the lowest $L_{\text {peak }}$ values. The obtained mean value is $\sim 0.05$, which is in excellent agreement with the calculations of Cornilleau-Wehrlin et al. (1985). These results are also very consistent with observations from Cluster (Parrot et al., 2003, 2004) and THEMIS spacecraft (Agapitov et al., 2011b), since the power of the reflected signals was found to be $0.005-0.02$ and $0.025-0.05$ of the source signal, respectively.

\section{Discussion and conclusions}

Lower-band chorus wave emissions, generated at the magnetic equator, are propagated into the Earth's inner magnetosphere by means of ray tracing technique (see Breuillard et al., 2012b,a, for details). After eventually undergoing the LHR reflection at higher latitudes, the trajectories of the reflected rays are processed to analyze their geometrical spreading back into the equatorial plane. We study the geometrical spreading as a function of the wave initial parameters $\left(R_{0}, \omega, \theta_{0}, \varphi_{0}\right)$, which allows us to determine the wave power distribution of reflected chorus waves after their first reflection at high latitude. We notably show that the spatial dispersion of reflected chorus waves is large, especially for the lower frequency waves with low $\theta_{0}$, for which it can reach several $R_{\mathrm{E}}$ in both radial and azimuthal distances, towards larger $L$ shells.

The impact of spatial spreading of waves can be important for wave-particle resonant interaction. Figure 1 for instance shows that localized wave source around $L \sim 6-7$ should produce relatively wide wave distribution at $L \sim 5$ up to $L \sim 10$. This localized source can mimic wave generation by transient particle injections into the inner magnetosphere (such injections often stop around $L \sim 6-7$ and can penetrate up to the plasmapause; see Ingraham et al., 2001; Dubyagin et al., 2011, and references therein). Our simulations demonstrate that waves generated in a localized source fill a wide region due to the divergence of their trajectories. Thus, the potential region of wave-particle resonant interaction can be substantially larger than the initial region where the flow of injected anisotropic plasma stops. As a result, even smallscale injections can be responsible for wave-particle resonant interaction in large spatial domains, corresponding to large regions of particle precipitation (see, e.g., Paschmann et al., 2002).

In order to compare our simulations with spacecraft data, we then model discrete chorus elements taking as a basis typically observed chorus properties at the equator. We show the reflected wave power distribution as a function of $L$ shell, for different distances of SCE generation, as well as the ratio of waves of the initial SCE that actually reach the equatorial plane. This allows us to calculate the attenuation ratio of reflected wave power along the $L$ shell values. The ratio is found to be about $0.005-0.06$ with a mean value of $\approx 0.05$, which is very consistent with theoretical calculations (e.g., Cornilleau-Wehrlin et al., 1985) and recent Cluster (Parrot et al., 2003, 2004) and THEMIS (Agapitov et al., 2011b) observations.

The study of the characteristics of the rays' spatial dispersion by means of ray tracing allows us to calculate the attenuation of reflected chorus wave power in every position in the inner magnetosphere. The large spreading of such a set of rays shows that chorus waves, even generated from a localized source at the equator, can fulfill large regions of the inner magnetosphere where wave-particle interactions thus occur. Determining the effects of such waves on energetic particles in these regions could enhance the understanding of inner magnetospheric dynamics. These effects could be important notably at higher latitudes, where the reflected wave power is comparable to its source signal.

Moreover, we observe that for waves generated at lower radial distances the wave power is less spread in $L$ shells and more focused close to the plasmapause, yet very few waves can penetrate into the plasmasphere. This interesting result seems contradictory to the results of Bortnik et al. (2009, 2011), although this can be due to the chosen plasma density parameters, and notably the level of geomagnetic activity (here $\mathrm{Kp}=4$ ), which defines the shape of the plasmapause. A sharp plasmapause could thus prevent the penetration of reflected chorus waves in the plasmasphere (Wang et al., 2011). However this aspect needs more detailed discussion and will be addressed in future work.

Acknowledgements. This work was supported by CNES through the grant Modèles d'ondes. The work of V. Krasnoselskikh and A. Artemyev was also partially supported by project 8527 of the Ministry of Education and Science of the Russian Federation.

Guest Editor M. Balikhin thanks S. Bourdarie and three anonymous referees for their help in evaluating this paper. 
The publication of this article is financed by CNRS-INSU.

\section{References}

Agapitov, O., Krasnoselskikh, V., Khotyaintsev, Y. V., and Rolland, G.: A statistical study of the propagation characteristics of whistler waves observed by Cluster, Geophys. Res. Lett., 38, L20103, doi:10.1029/2011GL049597, 2011a.

Agapitov, O., Krasnoselskikh, V., Zaliznyak, Yu., Angelopoulos, V., Le Contel, O., and Rolland, G.: Observations and modeling of forward and reflected chorus waves captured by THEMIS, Ann. Geophys., 29, 541-550, doi:10.5194/angeo-29-541-2011, $2011 \mathrm{~b}$.

Agapitov, O., Krasnoselskikh, V., Khotyaintsev, Y. V., and Rolland, G.: Correction to "A statistical study of the propagation characteristics of whistler waves observed by Cluster", Geophys. Res. Lett., 39, L24102, doi:10.1029/2012GL054320, 2012.

Agapitov, O., Artemyev, A., Krasnoselskikh, V., Khotyaintsev, Y. V., Mourenas, D., Breuillard, H., Balikhin, M., and Rolland, G.: Statistics of whistler-mode waves in the outer radiation belt: Cluster STAFF-SA measurements, J. Geophys. Res. Space Physics, 118, 3407-3420, doi:10.1002/jgra.50312, 2013.

Bortnik, J., Thorne, R. M., and Meredith, N. P.: The unexpected origin of plasmaspheric hiss from discrete chorus emissions, Nature, 452, 62-66, doi:10.1038/nature06741, 2008.

Bortnik, J., Li, W., Thorne, R. M., Angelopoulos, V., Cully, C., Bonnell, J., Le Contel, O., and Roux, A.: An Observation Linking the Origin of Plasmaspheric Hiss to Discrete Chorus Emissions, Science, 324, 775, doi:10.1126/science.1171273, 2009.

Bortnik, J., Chen, L., Li, W., Thorne, R. M., and Horne, R. B.: Modeling the evolution of chorus waves into plasmaspheric hiss, J. Geophys. Res. (Space Physics), 116, A08221, doi:10.1029/2011JA016499, 2011.

Breuillard, H., Mendzhul, D., and Agapitov, O.: Effects of equatorial chorus wave normal azimuthal distribution on wave propagation, Adv. Astron. Space Phys., 2, 167-172, 2012a.

Breuillard, H., Zaliznyak, Y., Krasnoselskikh, V., Agapitov, O., Artemyev, A., and Rolland, G.: Chorus wave-normal statistics in the Earth's radiation belts from ray tracing technique, Ann. Geophys., 30, 1223-1233, doi:10.5194/angeo-30-1223-2012, 2012 b.

Burtis, W. J. and Helliwell, R. A.: Banded chorus - A new type of VLF radiation observed in the magnetosphere by OGO 1 and OGO 3, J. Geophys. Res., 74, 3002-3010, doi:10.1029/JA074i011p03002, 1969.

Burtis, W. J. and Helliwell, R. A.: Magnetospheric chorus - Occurrence patterns and normalized frequency, Planet. Space Sci., 24, 1007-1024, doi:10.1016/0032-0633(76)90119-7, 1976.

Burton, R. K. and Holzer, R. E.: The Origin and Propagation of Chorus in the Outer Magnetosphere, J. Geophys. Res., 79, 10141023, doi:10.1029/JA079i007p01014, 1974.

Chum, J. and Santolík, O.: Propagation of whistler-mode chorus to low altitudes: divergent ray trajectories and ground accessibility, Ann. Geophys., 23, 3727-3738, doi:10.5194/angeo-23-37272005, 2005.
Cornilleau-Wehrlin, N., Solomon, J., Korth, A., and Kremser, G.: Experimental study of the relationship between energetic electrons and ELF waves observed on board GEOS - A support to quasi-linear theory, J. Geophys. Res., 90, 4141-4154, doi:10.1029/JA090iA05p04141, 1985.

Dubyagin, S., Sergeev, V., Apatenkov, S., Angelopoulos, V., Runov, A., Nakamura, R., Baumjohann, W., McFadden, J., and Larson, D.: Can flow bursts penetrate into the inner magnetosphere?, Geophys. Res. Lett., 38, L08102, doi:10.1029/2011GL047016, 2011.

Edgar, B. C. and Smith, R. L.: (Abstract) Fall URSI Meeting, Ann Arbor, Michigan, 1967.

Gallagher, D. L., Craven, P. D., and Comfort, R. H.: Global core plasma model, J. Geophys. Res., 105, 18819-18834, doi:10.1029/1999JA000241, 2000.

Haque, N., Spasojevic, M., Santolík, O., and Inan, U. S.: Wave normal angles of magnetospheric chorus emissions observed on the Polar spacecraft, J. Geophys. Res., 115, A00F07, doi:10.1029/2009JA014717, 2010.

Hayakawa, M., Parrot, M., and Lefeuvre, F.: The wave normals of ELF hiss emissions observed onboard GEOS 1 at the equatorial and off-equatorial regions of the plasmasphere, J. Geophys. Res., 91, 7989-7999, doi:10.1029/JA091iA07p07989, 1986.

Hikishima, M., Omura, Y., and Summers, D.: Microburst precipitation of energetic electrons associated with chorus wave generation, Geophys. Res. Lett., 37, L07103, doi:10.1029/2010GL042678, 2010.

Hines, C. O.: Heavy-ion effects in audio-frequency radio propagation, J. Atmospheric Terrest. Phys., 11, 36-42, 1957.

Horne, R. B., Thorne, R. M., Shprits, Y. Y., Meredith, N. P., Glauert, S. A., Smith, A. J., Kanekal, S. G., Baker, D. N., Engebretson, M. J., Posch, J. L., Spasojevic, M., Inan, U. S., Pickett, J. S., and Decreau, P. M. E.: Wave acceleration of electrons in the Van Allen radiation belts, Nature, 437, 227-230, doi:10.1038/nature03939, 2005.

Ingraham, J. C., Cayton, T. E., Belian, R. D., Christensen, R. A., Friedel, R. H. W., Meier, M. M., Reeves, G. D., and Tuszewski, M.: Substorm injection of relativistic electrons to geosynchronous orbit during the great magnetic storm of March 24, 1991, J. Geophys. Res., 106, 25759-25776, doi:10.1029/2000JA000458, 2001.

Jirícek, F., Shklyar, D. R., and Třiska, P.: LHR effects in nonducted whistler propagation - new observations and numerical modelling, Ann. Geophys., 19, 147-157, doi:10.5194/angeo-19-1472001, 2001.

Kimura, I.: Effects of ions on whistler-mode raytracing, Radio Sci., 1, 269-283, 1966.

LeDocq, M. J., Gurnett, D. A., and Hospodarsky, G. B.: Chorus Source Locations from VLF Poynting Flux Measurements with the Polar Spacecraft, Geophys. Res. Lett., 25, 4063, doi:10.1029/1998GL900071, 1998.

Li, W., Thorne, R. M., Angelopoulos, V., Bortnik, J., Cully, C. M., Ni, B., LeContel, O., Roux, A., Auster, U., and Magnes, W.: Global distribution of whistler-mode chorus waves observed on the THEMIS spacecraft, Geophys. Res. Lett., 36, L09104, doi:10.1029/2009GL037595, 2009.

Li, W., Bortnik, J., Thorne, R. M., and Angelopoulos, V.: Global distribution of wave amplitudes and wave normal angles of chorus waves using THEMIS wave observations, J. Geophys. Res., 
116, A12205, doi:10.1029/2011JA017035, 2011.

Lyons, L. R. and Thorne, R. M.: The magnetospheric reflection of whistlers, Planet. Space Sci., 18, 1753-1767, doi:10.1016/00320633(70)90009-7, 1970.

Lyons, L. R. and Thorne, R. M.: Equilibrium Structure of Radiation Belt Electrons, J. Geophys. Res., 78, 2142-2149, doi:10.1029/JA078i013p02142, 1973.

Meredith, N. P., Horne, R. B., and Anderson, R. R.: Substorm dependence of chorus amplitudes: Implications for the acceleration of electrons to relativistic energies, J. Geophys. Res., 106, 13165-13178, doi:10.1029/2000JA900156, 2001.

Meredith, N. P., Cain, M., Horne, R. B., Thorne, R. M., Summers, D., and Anderson, R. R.: Evidence for chorus-driven electron acceleration to relativistic energies from a survey of geomagnetically disturbed periods, J. Geophys. Res., 108, 1248, doi:10.1029/2002JA009764, 2003.

Meredith, N. P., Horne, R. B., Sicard-Piet, A., Boscher, D., Yearby, K. H., Li, W., and Thorne, R. M.: Global model of lower band and upper band chorus from multiple satellite observations, J. Geophys. Res. (Space Physics), 117, A10225, doi:10.1029/2012JA017978, 2012.

Olson, W. P. and Pfitzer, K. A.: Magnetospheric magnetic field modeling, Tech. rep., 1977.

Parrot, M., Santolík, O., Cornilleau-Wehrlin, N., Maksimovic, M., and Harvey, C.: Magnetospherically reflected chorus waves revealed by ray tracing with CLUSTER data, Ann. Geophys., 21, 1111-1120, doi:10.5194/angeo-21-1111-2003, 2003.

Parrot, M., Santolík, O., Gurnett, D. A., Pickett, J. S., and Cornilleau-Wehrlin, N.: Characteristics of magnetospherically reflected chorus waves observed by CLUSTER, Ann. Geophys., 22, 2597-2606, doi:10.5194/angeo-22-2597-2004, 2004.

Paschmann, G., Haaland, S., and Treumann, R.: Auroral plasma physics, Space Sci. Rev., 103, 1-477, doi:10.1023/A:1023030716698, 2002.

Santolík, O., Gurnett, D. A., Pickett, J. S., Parrot, M., and Cornilleau-Wehrlin, N.: Central position of the source region of storm-time chorus, Planet. Space Sci., 53, 299-305, doi:10.1016/j.pss.2004.09.056, 2005a.

Santolík, O., Macúšová, E., Yearby, K. H., Cornilleau-Wehrlin, N., and Alleyne, H. StC. K.: Radial variation of whistler-mode chorus: first results from the STAFF/DWP instrument on board the Double Star TC-1 spacecraft, Ann. Geophys., 23, 2937-2942, doi:10.5194/angeo-23-2937-2005, 2005b.

Santolík, O., Gurnett, D. A., Pickett, J. S., Grimald, S., Décreau, P. M. E., Parrot, M., Cornilleau-Wehrlin, N., El-Lemdani Mazouz, F., Schriver, D., Meredith, N. P., and Fazakerley, A.: Wave-particle interactions in the equatorial source region of whistler-mode emissions, J. Geophys. Rese. (Space Physics), 115, A00F16, doi:10.1029/2009JA015218, 2010.
Sazhin, S. S. and Hayakawa, M.: Magnetospheric chorus emissions - A review, Planet. Space Sci., 40, 681-697, doi:10.1016/00320633(92)90009-D, 1992.

Shklyar, D. R., Chum, J., and Jiříček, F.: Characteristic properties of $\mathrm{Nu}$ whistlers as inferred from observations and numerical modelling, Ann. Geophys., 22, 3589-3606, doi:10.5194/angeo-223589-2004, 2004.

Shprits, Y. Y., Subbotin, D. A., Meredith, N. P., and Elkington, S. R.: Review of modeling of losses and sources of relativistic electrons in the outer radiation belt II: Local acceleration and loss, J. Atmos. Solar-Terr. Phys., 70, 1694-1713, doi:10.1016/j.jastp.2008.06.014, 2008.

Smith, R. L. and Angerami, J. J.: Magnetospheric Properties Deduced from OGO 1 Observations of Ducted and Nonducted Whistlers, J. Geophys. Res., 73, 1, doi:10.1029/JA073i001p00001, 1968.

Suchy, K.: Real Hamilton equations of geometric optics for media with moderate absorption, Radio Sci., 16, 1179-1182, doi:10.1029/RS016i006p01179, 1981.

Summers, D., Ma, C., Meredith, N. P., Horne, R. B., Thorne, R. M., Heynderickx, D., and Anderson, R. R.: Model of the energization of outer-zone electrons by whistler-mode chorus during the October 9, 1990 geomagnetic storm, Geophys. Res. Lett., 29, 2174, doi:10.1029/2002GL016039, 2002.

Summers, D., Ni, B., and Meredith, N. P.: Timescales for radiation belt electron acceleration and loss due to resonant wave-particle interactions: 2. Evaluation for VLF chorus, ELF hiss, and electromagnetic ion cyclotron waves, J. Geophys. Res., 112, A04207, doi:10.1029/2006JA011993, 2007.

Thorne, R. M. and Kennel, C. F.: Quasi-Trapped VLF Propagation in the Outer Magnetosphere, J. Geophys. Res., 72, 857, doi:10.1029/JZ072i003p00857, 1967.

Trakhtengerts, V. Y. and Rycroft, M. J.: Whistler and Alfvén Mode Cyclotron Masers in Space, Cambridge University Press, 2008.

Tsurutani, B. T. and Smith, E. J.: Two types of magnetospheric ELF chorus and their substorm dependences, J. Geophys. Res., 82, 5112-5128, doi:10.1029/JA082i032p05112, 1977.

Wang, C., Zong, Q., Xiao, F., Su, Z., Wang, Y., and Yue, C.: The relations between magnetospheric chorus and hiss inside and outside the plasmasphere boundary layer: Cluster observation, J. Geophys. Res. (Space Physics), 116, A07221, doi:10.1029/2010JA016240, 2011. 